\title{
Evaluation of Monilinia fructicola on apricot fruits
}

\section{J. Del Cueto', P. Stefani², D. Christen ${ }^{1}$}

${ }^{1}$ Agroscope Changins-Wädenswil Research Station ACW, Schloss, P.O. Box 185, 8820 Wädenswil, Switzerland.

2Department of Extension, Training and Communication, FiBL, Ackerstrasse $113 \mathrm{CH}-5070$ Frick, Switzerland

jorge-luis.del-cueto-chocano@agroscope.admin.ch

\section{State of the art}

Monilinia fructicola has been a quarantine pathogen in Europe until 2014; however, the disease risk remains large for Prunus species, because of the continuing spreading around Europe. In order to assess the impact of this fungus on apricot fruits, differences in variety susceptibility need to be evaluated.
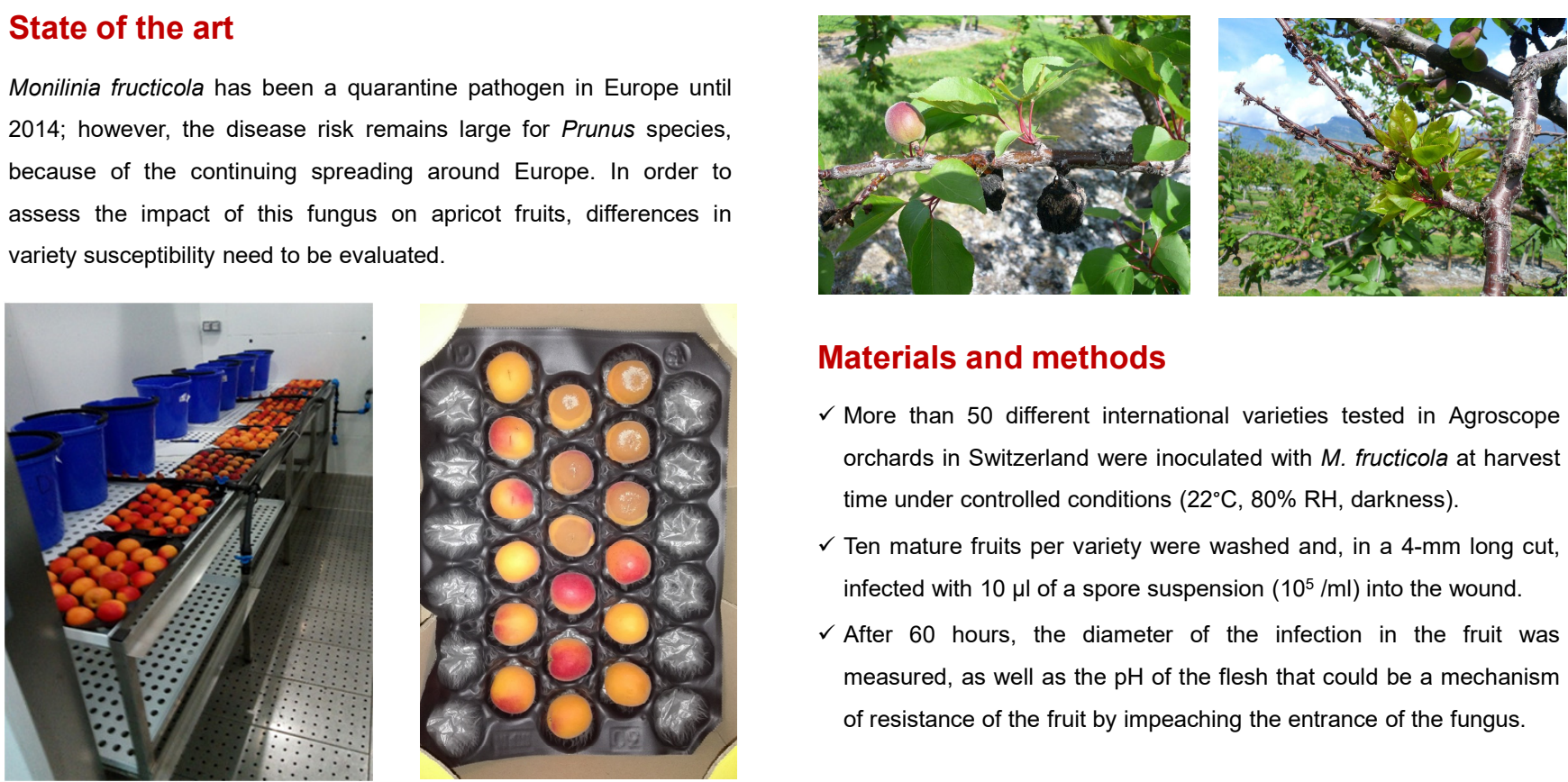

Materials and methods

$\checkmark$ More than 50 different international varieties tested in Agroscope orchards in Switzerland were inoculated with M. fructicola at harvest time under controlled conditions $\left(22^{\circ} \mathrm{C}, 80 \% \mathrm{RH}\right.$, darkness $)$.

$\checkmark$ Ten mature fruits per variety were washed and, in a 4-mm long cut, infected with $10 \mu \mathrm{l}$ of a spore suspension $\left(10^{5} / \mathrm{ml}\right)$ into the wound.

$\checkmark$ After 60 hours, the diameter of the infection in the fruit was measured, as well as the $\mathrm{pH}$ of the flesh that could be a mechanism of resistance of the fruit by impeaching the entrance of the fungus.
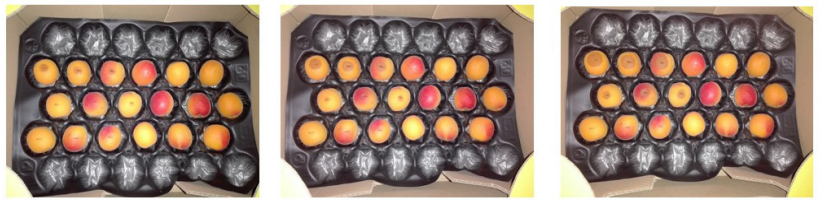

The most susceptible varieties were Big Red, Vanilla cot, Bigaly

$\checkmark$ No good correlation between branches and flowers symptoms and fruit symptoms was found, corroborating the fact that both infections are independent of each other.

$\checkmark$ Furthermore, no link between the levels of $M$. fructicola infection
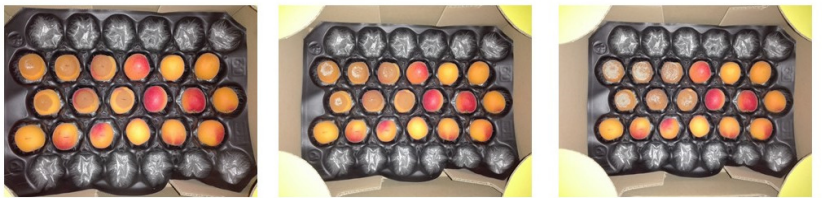

45,0
40,0
35,0
30,0
25,0
20,0
15,0
10,0
5,0
0,0

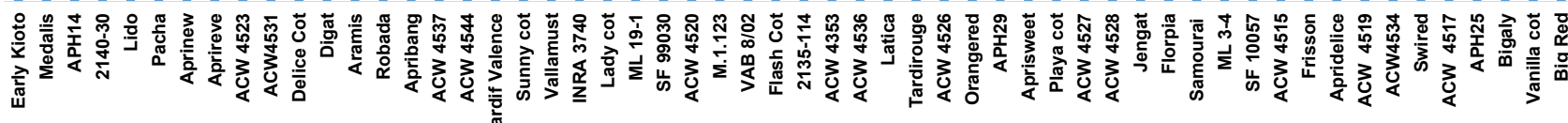

Fruit infection diameter (in $\mathrm{mm}$ ) under controlled conditions in different varieties after inoculation with spores of $M$. fructicola under controlled conditions. Orange line indicates $\mathrm{pH}$ of the flesh.

d象

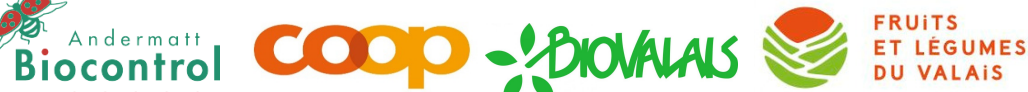

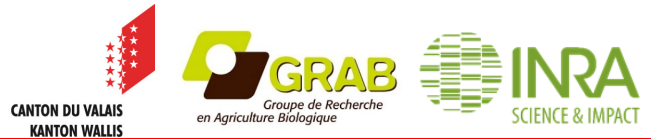

